\title{
Antibacterial and Wound Healing Activities of Silver Nanoparticles Embedded in Cellulose Compared to Other Polysaccharides and Protein Polymers
}

Mehran Alavi ( $\square$ mehranbio83@gmail.com )

Razi University of Kermanshah: Razi University https://orcid.org/0000-0002-5691-8326

Rajender S. Varma

Palacky University

\section{Research Article}

Keywords: AgNPs, natural polymers, biocompatibility, antibacterial activities, infected chronic wounds, wound dressing

Posted Date: April 30th, 2021

DOI: https://doi.org/10.21203/rs.3.rs-404394/v1

License: (c) (i) This work is licensed under a Creative Commons Attribution 4.0 International License. Read Full License

Version of Record: A version of this preprint was published at Cellulose on July 12th, 2021. See the published version at https://doi.org/10.1007/s10570-021-04067-3. 


\section{Abstract}

The aggregation of silver nanoparticles (AgNPs) in colloidal solution and the oxidative cytotoxicity towards human cells are two major hindrances for their thriving medicinal applications. Their incorporation in natural polymers such as cellulose, chitosan, alginate, collagen, gelatin, silk fibroin, carrageenan, hyaluronic acid, keratin and starch may be an alluring alternative strategy to sidestep these complications and attaining the advantageous wound dressings. Biocompatibility, bioavailability, biodegradability, and inherent therapeutic properties known for theses polymers, would accelerate the healing of infected chronic wounds. However, the low thermal stability, mechanical strength, rapid biodegradation, and weak washing resistance properties are some of the limitations for these polymers. Herein, recent advances, present challenges and future perspective for AgNPs incorporated nanocomposites (NCs) are discussed to realize ideal antibacterial activities by exploiting the abundant natural biopolymers.

\section{Introduction}

Hemostasis, inflammation, proliferation, and tissue remodeling are four main continuous phases of wound healing, which can be slowed by bacterial and fungal infections particularly in the case of diabetic foot ulcers (DFUs) (Ezhilarasu et al. 2020). Multidrug resistant (MDR) bacteria in infected wounds can complicate this situation by their adaptation mechanisms to a wide range of conventional antibiotics. Moreover, bacterial biofilms with complex and sessile community of bacteria and fungi embedded in extracellular matrix (ECM) composed mainly of exopolysaccharides (EPS) are more resistant to penetration of antibiotics (Pouget et al. 2020). Silver nanoparticles (AgNPs) have appropriate bacteriostatic and bactericidal traits but are cytotoxicity towards eukaryotic cells and hence their limited therapeutic applications. Micro- and nanoformulations based on natural polymers with appropriate biocompatibility, bioavailability, biodegradability, and therapeutic attributes can reduce the side effects of AgNPs under physiological conditions. Antibacterial mechanisms of AgNPs have been studied via the interaction of $\mathrm{Ag}^{+}$with cell wall, membrane, and enzymes of the respiratory chain, proteins and nucleic acids of bacteria. Reactive oxygen species (ROS) such as superoxide $\left({ }^{\circ} \mathrm{O}_{2}{ }^{-}\right)$and hydroxyl radical ('OH) can ensue from the reaction of $\mathrm{Ag}^{+}$ions with molecular oxygen in outside and inside medium of bacteria (Alavi et al. 2019; Taran et al. 2016; Alavi and Karimi 2019, 2020).

Living organisms encompassing animals, plants, bacteria, fungi, and alga can synthesize natural polymers or biopolymers with higher biocompatibility, bioavailability, and biodegradability properties. These polymers may be classified based on their monomers, wherein polysaccharide, polypeptide, and polynucleotides are three major forms comprising glucose, amino acids, and nucleotides. Among the natural polymers, cellulose, chitosan, alginate, collagen, gelatin, silk fibroin, hyaluronic acid, keratin, carrageenan, and starch have garnered more attention owing to their unique physicochemical and biological properties (Kaczmarek et al. 2020). 


\section{Polysaccharide Polymers}

\section{Cellulose}

Three major properties namely biocompatibility, biodegradability, and appropriate hydrophobicity are indicated for cellulose polymer. As forms of nano scale, cellulose nanocrystal (CNC) and cellulose nanofibril (CNF) can be prepared commonly by acid hydrolysis and high pressure homogenization (Alavi 2019). These nanomaterials (NMs) are employed to improve the mechanical strength of hydrogel, cryogels, and scaffold (Abdelgawad et al. 2020). Functionalization of cellulose can be completed by targeting its hydroxyl groups to improve mechanical strength, biochemical reactivity, and absorbency in physiochemical conditions. AgNPs are often synthesized via the reduction of $\mathrm{Ag}^{+}$ions and stabilizing of ensuing NPs by hydroxyl groups of this polymer without any additional stabilizers (Alavi and Nokhodchi 2020). Sustained release of $\mathrm{Ag}^{+}$ions is critical to hinder the growth of MDR bacteria and formation of biofilm at wound site. Ag nanowires (AgNWs) with higher aspect ratio (length to diameter ratio) can improve the release of $\mathrm{Ag}^{+}$ions and mechanical properties of wound dressings. Stretchable wound dressing has been made combining the bacterial cellulose (BC) with AgNWs in three volume ratios of 10 : 4.5, $10: 3$, and $10: 1$, wherein after $24 \mathrm{~h}$, sustained release of $\mathrm{Ag}^{+}$ions was observed at the period 9 days for all volume ratios; highest tensile stress and Young's module were $1.56 \mathrm{MPa}$ and $2.88 \mathrm{GPa}$, respectively for the volume ratio of $10: 4.5$. Additionally, the complete wound closure after 12 days as well as significant antibacterial effect $(\approx 100 \%$ after $6 \mathrm{~h}$ ) against $E$. coli and $S$. aureus bacteria were indicated for this volume ratio (Wan et al. 2020). Wound dressings based on polymeric hydrogels encompassing AgNPs are interesting alternative to enhance the antibacterial activity with wound healing properties in vitro and in vivo. AgNPs have been loaded on hydrogel composed of cellulose carbamate using Tween 80 , cetyl trimethylammonium bromide (CTAB) and rarasaponin as values of $7.53,9.94$ and $10.15 \mathrm{mg} / \mathrm{g}$, sequentially. Compared to other groups, higher antibacterial effect was indicated for AgNPs $(99.4 \mu \mathrm{g})$ @hydrogel-CTAB as inhibition zone diameters (IZDs) of 18.98 and $19.84 \mathrm{~mm}$ toward E. coli and $S$. aureus, which may be caused by synergistic effect of AgNPs with cellulose carbamate and CTAB surfactant. It is worth noting that CTAB has positive charge with high affinity for the negative charge of cell membranes (Bundjaja et al. 2021). Stability and durability of AgNPs is critical factor for employing these NPs in a large-scale production. There are various derivatives for cellulose such as hydroxypropyl methylcellulose

and ethylcellulose, which can contribute in synthesis and stabilization of AgNPs at 25 and $4.0^{\circ} \mathrm{C}$ for long period ( 3 months). Antibacterial activity of four types of AgNPs involving bare AgNPs, ethylcellulose (EC)-AgNPs, hydroxypropyl methylcellulose (HPMC)-AgNPs, methylcellulose (MC)-AgNPs and polyethylene glycol ( $\left.\mathrm{PEG}_{6000}\right)$-AgNPs toward E. coli and $S$. aureus were evaluated in a comparative way. Internalization of HPMC-AgNPs > EC-AgNPs > MC-AgNPs formulations into bacteria was higher compared to PEG-AgNPs and pure AgNPs for both bacteria significantly E. coli (Figure 1). Higher penetration ability of HPMC, EC, and MC into the bacterial cell membrane with phospholipid structure, more NPs production by these cellulose derivatives, and sustained release of silver ions may be reasons for this difference (Abdellatif et al. 2021). 


\section{Chitosan}

The linear polymer polysaccharide containing D-glucosamine and $N$-acetyl-D-glucosamine by $\beta$ - $(1 \rightarrow 4)$ linkage, is obtained from the chitin shells of shrimp and other crustaceans such as crabs and lobsters by alkaline treatment (Alavi and Rai 2019). Suitably-designed scaffold with large pores size $(\geq 250 \mu \mathrm{m})$ promote the adherence, proliferation and migration of skin cells which leads to rapid regeneration of dermal tissue. Further, chitosan $\left(\mathrm{C}_{56} \mathrm{H}_{103} \mathrm{~N}_{9} \mathrm{O}_{39}\right)$ with amine groups can interact with negative charge of cell envelope of bacteria and synergize the antibacterial activity of AgNPs. Wound dressing comprising chitosan-collagen-AgNPs (spherical by size in the range of $10-25 \mathrm{~nm}$ at concentration of $10 \mu \mathrm{g} / \mathrm{mL}$ ) illustrated accelerated wound healing via promotion of the fibroblasts migration, more expression of asmooth muscle actin (a-SMA), and macrophage performance (Figure 2). Dead cells at wound site can be eliminated by increasing the number of IL-10 levels and M2 macrophages in inflammatory phase (J. Wang and Xu 2020). In another study, a faster wound healing by abundant granulation tissue, higher collagen formation, lower levels of macrophage and vessel density were indicated for the electrospun chitosan-based NFs containing AgNPs at concentrations of 12 and $60 \mathrm{mg}$ in comparison with control samples after 14 days of treatment. Moreover, this investigation demonstrated that the inorganic ions and proteins can slow down and block the release of $\mathrm{Ag}^{+}$from AgNPs (Shao et al. 2019).

\section{Alginate}

Brown alga such as Turbinaria turbinata, Sargassum filipendula, Macrocystis pyrifera,Saccharina longicruris, Sargassum carpophyllum, and Sargassum siliquosum as well as bacteria namely $P$. aeruginosa have the ability to produce hydrophilic polymer of alginate containing three types of block structure: G block (poly a-L-guluronic acid), M block (ß-D-mannuronic acid), and MG block (both polyuronic acids) with $\beta$-(1-4) glycosidic linkage (1-4) (García-Ríos et al. 2012; Valentine et al. 2020). AgNPs have been incorporated in the matrix of sodium alginate and collagen via interactions between the amine group of collagens and the carboxylate group of alginates with AgNPs. In this way, $\mathrm{NaBH}_{4}$ and polyvinyl pyrrolidone (PVP) were employed, respectively as reducing and stabilizing agents to obtain spherical AgNPs with the mean particle size of $7 \mathrm{~nm}$. AgNPs in concentration of $50 \mu \mathrm{M}$ exhibited negligible cytotoxicity against $\mathrm{NIH} 3 \mathrm{~T} 3$ cell line with bacterial inhibition toward $E$. coli and $S$. aureus as inhibition zone diameters (IZDs) of 2.7 and $1.9 \mathrm{~mm}$, respectively (Zhang et al. 2018). As an efficient strategy, polydopamine (PDA) spheres (a mean diameter of $0.430 \mu \mathrm{m}$ ) were decorated by AgNPs (size in the range of $50-70 \mathrm{~nm}$ ) followed by immobilization on oxidized sodium alginate (OSA) wound dressing. Significant porosity value of $77.30 \%$ (relative to hyaluronic acid/cationized dextran (73\%) and quercetin/duck's feet collagen/hydroxyapatite $(76.36 \%)$ ) and blood compatibility at concentration of 200 ppm made this NC ideal as a safe wound dressing (Liang et al. 2020).

\section{Gelatin}

In addition to enzymatic extraction (Ahmad et al. 2017), partial acid and alkaline hydrolysis of type I collagen from the skin, bones, cartilage, and meat can produce gelatin types A and B, respectively (Yang 
et al. 2019). Gelatin as an extracellular matrix (ECM) protein may be used to prepare drug and gene delivery systems, wound dressings, tissue engineering, and 3D cell culture (Han and Lv 2019; Afewerki et al. 2019). PVP-stabilized AgNPs have been aminated via 3-aminopropyltriethoxysilane (APTES) treatment at $70{ }^{\circ} \mathrm{C}$ for $12 \mathrm{~h}$ and incorporated in the carboxylated CNF to achieve injectable nanoformulation for wound therapy in nursing infants. This formulation was employed to accelerate inflammation and proliferation phases of wound healing by increasing the number of white blood cells (WBCs) specifically neutrophils at wound site after 14 days of treatment compared to control group of CNF/gelatin (Gou et al. 2020). It is noteworthy that in the inflammation phase, increased capillary permeability and migration of cells particularly neutrophils to the wound tissue would help sterilize the wounds and release proteases to remove the denatured ECM followed by transformation of monocytes into macrophages in the wound site, regulated by cytokines such as monocyte chemotactic protein-1 (MCP-1) and transforming growth factor- $\beta$ (TGF- $\beta$ ) (J. Wang and Xu 2020). Wound healing activities for combination of metal NPs (MNPs)/metal oxide NPs (MONPs) with important growth factors (GFs) involving fibroblast growth factor (FGF), epidermal growth factor (EGF), bone morphogenic proteins (BMPs), TGF- $\beta$, vascular endothelial growth factor (VEGF), and platelet-derived growth factor (PDGF) in wounds' healing phases of hemostasis, inflammation, proliferation, and remodeling/maturation have been reviewed previously (Alavi and Rai 2020). Incorporation of AgNPs (0.004 M) in hydrogel formulated as sodium alginate/gelatin in the ratio of 80:20 displayed prowess for the topical application with MIC values toward $P$. aeruginosa ( 0.5 $\mathrm{ppm}$ ) and $S$. aureus (53 ppm), higher consistency and a significant epithelialization after 14 days treatment compared to control and hydrogels without AgNPs (Figure 3) (Diniz et al. 2020).

\section{Carrageenan (CAR)}

Three types of CAR including kappa, iota, and lambda are commercial polymers, which are commonly deployed in food and pharmacy industries. The stabilization of MNPs/MONPs, gelling and thickening applications, specifically in food industry, have been reported for CAR as a sulfated polymer of galactose units linked alternatively by $\beta-1,4$ and a-1,3 (Pandey et al. 2020). CAR and carboxymethyl CNC were used to formulate cryogel loaded AgNPs with $100 \%$ reduction ability towards $E$. coli and $S$. aureus. This polymeric composition instigated desirable controlled and sustained release by $40 \mu \mathrm{g} / \mathrm{mL}$ of AgNPs within 250 min. rapid wound healing; suitable mechanical strength of this wound dressing originated from CAR and carboxymethyl CNC polymers, respectively (Abdelgawad et al. 2020). In addition to antiplanktonic and anti-biofilm activities towards $P$. aeruginosa and $S$. aureus, the stability of CAR-Ag NPs was indicated for 6 months in a colloidal medium which is due to the suitable interaction of CARs by their hydroxyl (-OH) groups with AgNPs (Goel et al. 2019).

\section{Hyaluronic acid (HA)}

$\mathrm{HA}$, hyaluronate or hyaluronan, is anionic polymer of nonsulafted glycosaminoglycan (disaccharides of $\mathrm{N}$-acetyl-D-glucosamine and D-glucuronic acid) with high molecular weight, and is present in prokaryotic and eukaryotic cells particularly in ECM section with the critical role in proliferation and migration of human cells in epithelial, neural, and connective tissues; inflammation response, angiogenesis and 
granulation of wounds are influenced positively by HA (Alemzadeh et al. 2020). According to molecular weight (MW) property, 1-25 × 104, 25-10 × 104, >1 ×106, > $6 \times 106$ Da were indicated for low MW, medium MW, high MW and very high MW of HA, respectively. All four stages of wound healing are influenced distinctly by low and high molecular weight of HA through interaction with the CD44 receptors related to granulocytes and monocytes as well as various interleukins such as IL-6, IL-1 $\beta$, and IL-8. (Graça et al. 2020). Wrapping bacteria, reactive oxygen species (ROS) production, lipid peroxidation, membrane disruption, interaction with glutathione (GSH), adenine, and proteins leakage were indicated as main antibacterial mechanisms for graphene oxide (GO) (Nanda et al. 2016; Mohammed et al. 2020). HAAgNPs NC was loaded on the GO sheet in solution of $N$-(3-Dimethylaminopropyl)- $N$-ethylcarbodiimide hydrochloride and $N$-hydroxysuccinimide. Bactericidal activity against $S$. aureus could be synergized by heat conversion ability of HA-AgNPs-GO, resulting from the higher specific surface area of GO, under near infrared (NIR) irradiation (808nm for $2 \mathrm{~min}$ ) from $25.5^{\circ} \mathrm{C}$ to $58.4^{\circ} \mathrm{C}$. Wound healing was effected by controlled release of $\mathrm{Ag}^{+}$ions from HA-AgNPs under hyaluronidase enzyme (Ran et al. 2017). Inflammatory step of wound healing can be affected negatively by ROS species, which can be controlled by suitable formulations of Ag and HA. Polygalacturonic acid (PGA), an herbal polymer, was employed as a reducing and stabilizer agent to prepare AgNPs followed by incorporation in nanofibers (NFs) comprising $\mathrm{HA}$ and poly vinyl alcohol (PVA) by electrospinning technique. Antibacterial activities were not observed for (PGA/HA)-PVA, while incorporation of AgNPs imparted significant antibacterial effects on $B$. subtilis, S. aureus, and E. coli. In addition, the percentages of wound contraction for (PGA/HA)-PVA and (AgNPs-PGA/HA)-PVA after 14 days were 97.42 and $99.51 \%$ relative to $92.43 \%$ for Garamycin $\AA$ cream as a control group (El-Aassar et al. 2020).

\section{Pectin}

Pectin, acidic hetropolysaccharides (linear chain of alpha (1-4) linked D-galacturonic acid; $\mathrm{C}_{6} \mathrm{H}_{10} \mathrm{O}_{7}$ ) with high molecular weight $(194.14 \mathrm{~g} / \mathrm{mol})$ are found in the cell walls of plant species. They are used as antioxidant, emulsifiers, food coloring agent, anti-infective agent, and stabilizers of NPs in the food and pharmaceutical industries (Mercado-Mercado et al. 2020; Jiang et al. 2020). Combination of pectin with other synthetic and natural polymers has been deployed as novel wound bandages to reduce the disadvantages of each individual polymer by itself. NFs comprising AgNPs/polyvinylalcohol (PVA)/polyvinylpyrrolydone (PVP)/pectin/mafenide acetate (MF) were electrospun in the weight ratio of 0.7 and $91.8 \mathrm{wt} \%$ for AgNPs and PVA as well as $2.5 \mathrm{wt} \%$ for PVP, pectin and MF, respectively. NPs were synthesized in PVA solution and mixed in solution of pectin, MF, and PVP followed by electrospinning on a grounded collecting drum. Higher antibacterial effect, although not adequate enough, was indicated against Gram-negative bacteria relative to Gram-positive bacteria owing to difference in thickness of bacterial cell wall. Moreover, the releases rate of AgNPs was influenced mainly by their interaction with the functional groups such as $-\mathrm{SO}_{2}$ and $-\mathrm{NH}_{2}$ of MF (Alipour et al. 2019). The size of AgNPs was affected by two parameters including weight percentage of pectin (extracted from citrus peel) $(0.5,1$, and $2 \%$ ) and temperature $\left(20\right.$ and $60^{\circ} \mathrm{C}$ ), wherein smaller diameter of NPs (8 $\mathrm{nm}$ ) was obtained for $1 \%$ of pectin in $60^{\circ} \mathrm{C}$. MIC values for pectin-AgNPs showed 31.25 and $500 \mu \mathrm{M}$ towards $E$. coli and $S$. 
epidermidis after 24 incubation compared to glutathione-AgNPs with MIC amounts of 140 and $1680 \mu \mathrm{M}$, respectively. In this regard, the ability of pectin to adhere to the bacterial membrane increases the antibacterial activity of AgNPs (Pallavicini et al. 2017).

\section{Starch}

Starch $\left(\mathrm{C}_{6} \mathrm{H}_{10} \mathrm{O}_{5}\right)_{n}$, the linear polymer of glucose as amylose and amylopectin in the branched form, is a renewable and biodegradable polysaccharide and has been applied for various applications such as drug delivery, micro and nano formulations of therapeutic agents, production of pharmaceutical tablets (Athira et al. 2018; Chen et al. 2020). This polymer has reducing and stabilizing functions in MNPs/MONPs synthesis in one-pot operation. Synthesized Ag/Au bimetallic NPs have been shown to affect the morphology change of $E$. coli as lacking flagella in treatment samples relative to control. $I_{50}$ values for these bimetallic NPs compared to AuNPs were $4.92 \pm 0.81$ and $6.95 \pm 1.70 \mu \mathrm{g} / \mathrm{mL}$ against multidrug resistant (MDR) E. coli and methicillin-resistant S. aureus (MRSA) (Lomelí-Marroquín et al. 2019). Isolated starch from babassu mesocarp, a by-product of babassu oil, was utilized to synthesize AgNPs with different diameter size of 124.2, 119.1, and $181.7 \mathrm{~nm}$ by microwave, autoclave, and water bath methods, respectively. In a comparative way, MBC value of $>27 \mu \mathrm{g} / \mathrm{mL}$ was determined for all NPs as well as $\mathrm{AgNO}_{3}$ salt against $S$. aureus. The prominent cell membrane damage, pore formation and the release of bacteria contents of $E$. coli was observed after treatment by AgNP prepared by water bath method (at sub value of MIC=6.75) (Bastos Araruna et al. 2020). Combination of AgNPs, starch, PVA, and GO as a scaffold showed synergic antibacterial effects with the Modulus and tensile strength of $145 \%$ and $26.81 \%$, respectively (Usman et al. 2016). In this regard, GO can increase the antibacterial activities of AgNPs by lipid peroxidation resulting from carbon radicals $\left({ }^{*} \mathrm{C}\right)$ and cellular membrane damage caused by sharp edge of GO (Alavi et al. 2020).

\section{Cyclodextrin (CD)}

Cyclodextrin (CD) is belongs to a cyclic oligosaccharides family with a macrocyclic ring of glucose subunits bonded by $a-1,4$ glycosidic links in three main types namely $6: a-C D, 7: \beta-C D$, and 8: $y-C D$, which can be used to encapsulate hydrophobic drugs in interior section due to improve its bioavailability, solubility, and stability. $a-C D$ and $y-C D$ s types are more water soluble relative to $\beta-C D$ (Tian et al. 2020). Carboxymethyl- $\beta$-cyclodextrin (CM- $\beta C D$ ) as a derivative of $C D$ has solubility of $50 \mathrm{mg} / \mathrm{mL}$, which can be used to load and stabilize AgNPs in various micro and nano formulations. Two concentrations of $\mathrm{CM}$ $\beta C D$ involving 50 and $25 \mathrm{mg}$ were reacted with chitosan $(50 \mathrm{mg})$, and glutaraldehyde $(0.013 \mathrm{mM})$ to produce hydrogels 1 and 2 ( $\mathrm{H} 1$ and $\mathrm{H} 2$ ). Incorporated constant amount of AgNPs in this formulation illustrated different antibacterial properties dependent on CM- $\beta C D$ concentration, wherein 19,15 , and 41.8 $\mathrm{mm}$ were observed for $\mathrm{H} 1$ and $\mathrm{H} 2$, and gentamycin $(30 \mu \mathrm{g})$ toward $E$. coli (Mohamadi Zahedi and Mansourpanah 2018). Gallic acid (GA) (3,4,5-trihydroxybenzoic acid), is a phenolic acid found in plants such as tea leaves, gallnuts, and oak bark and has antibacterial activities against Listeria monocytogenes, Pseudomonas aeruginosa, E. coli, and S. aureus by irreversible changes in membrane and cell wall morphology (Li et al. 2019). Synergic effect of GA and its antibacterial activity in hydrogel 
formulation was obtained by incorporation and loading of these materials in $\beta$-CD and GO, separately. Hydrogel film comprising PVA/GA@ßCD/Ag-GO demonstrated improved tensile strength (>126.2 MPa), sustained and slower GA release over $2 \mathrm{~h}$ and higher antibacterial activity towards $E$. coli and $S$. aureus compared to PVA/GA@ $\beta C D$ and PVA/ $\beta C D$ (Pooresmaeil and Namazi 2019). Hydroxypropyl- $\beta C D$ (HP$\beta C D)$ with solubility of $45 \%(w / v)$, as another derivative of $\beta-C D$, has been used as a delivery system to increase the solubility of therapeutic agents in aqueous media of biological conditions (Wei et al. 2017); 1 wt\% AgNPs in NFs of AgNPs-HP-BCD showed 11.6 and $10.7 \mathrm{~mm}$ for $S$. aureus and $E$. coli, respectively. Higher antibacterial capacity and The larger fiber diameter were observed for $2 \mathrm{wt} \%$ AgNPs in these NFs (Celebioglu et al. 2019). Thiomers, thiolated polymers, are polymers employed in micro and nanoformulations to augment the absorption of therapeutic agents in vaginal, eyes, mouth, and nose routes. Thiomers with low molecular mass can impart higher permeability and mucoadhesive properties to polymeric formulations (Palazzo et al. 2017). For instance, $\beta$-CD-SH1200 and $\beta$-CD-SH600 illustrated 46.37-and 39.73- fold higher mucoadhesion relative to $\beta$-CDs, respectively (Moghadam et al. 2018).

\section{Protein Polymers}

\section{Collagen}

Collagen has been found as the frequent protein in terrestrial and marine animals with desirable tensile strength, biocompatibility, bioavailability, and biodegradability properties for various therapeutic applications particularly tissue engineering (Lim et al. 2019; Divakar et al. 2019). Scaffold of collagen can attract and guide the migration of fibroblast cells along a connective tissue matrix (Dill and Mörgelin 2020). By interaction with blood platelets, this polymer can lead to the wound closure and prompt the acceleration of hemostatic phase of wound healing (Ding et al. 2020). However, weak washing resistance, low thermal stability, mechanical strength, and rapid biodegradation properties are some limitations for this protein (Pietrucha 2005; El-Fiqi et al. 2013). Obtaining stable colloidal solution of AgNPs with uniform size distribution may be realized by the appropriate selection of suitable reducing/stabilizing agents. Dialdehyde xanthan gum (DXG) with favorable biocompatibility was employed to produce spherical AgNPs in the range of 12-35 nm followed by incorporation in collagen matrix by cross linkage of DXG (via Schiff's base reaction) to attain the wound dressing. In addition to antibacterial activities toward $E$. coli, $S$. aureus, and $P$. aeruginosa, this sponge illustrated the complete integrity after physical pressing in contrast to collagen sponge. Lack of collagen in wound tissue was indicated for the collagen sponge and the sterile gauze compared to collagen-AgNPs NCs after a period of 18 days treatment (Ge et al. 2018).

\section{Keratin}

The main component of the wool, feather, horns, hair, and hooves is keratin, which has been investigated widely owing to its intrinsic physicochemical, mechanical durability, biocompatibility, and biological properties. In contrast to other biopolymers such as chitosan, collagen, cellulose, and starch, cumbersome chemical conditions are required to dissolve this polymer in water and organic solvents for 
appropriate extraction. The keratin extraction may be performed via the reduction, oxidation, microbial technique, microwave irradiation, and steam explosion pathways (Feroz et al. 2020). Based on mentioned advantages, a promising nanofibrous mat can be prepared from this type of polymer for apt wound bandages and tissue engineering (Esparza et al. 2017; Guo et al. 2019). For instance, a NC of keratin, polyurethane, and AgNPs can generate desirable antimicrobial impact. In this case, human hair was used as source of keratin followed by functionalization with iodoacetic acid to prepare $S$-(carboxymethyl) keratin. High concentration of AgNPs (5\% of silver nitrate) revealed more antibacterial effects against $E$. coli and $S$. aureus relative to polyurethane and polyurethane -S-(carboxymethyl) keratin (Y. Wang et al. 2016).

\section{Silk fibroin (SF) and sericin}

Various species of silk worm such as Bombyx mori can be used to extract SF (70-80\%) with cytocompatibility, high adhesiveness and suitable mechanical properties emanating from fibrillar structure with the $\beta$-sheet crystal of (Gly-Ser-Gly-Ala-Gly-Ala) , which can promote the adhesion of platelets and proliferation of stem cells (Patil et al. 2020; Anuduang et al. 2020). Anti-adhesion, antiplanktonic, and inhibition of biofilm was indicated for AgNPs/gentamycin-loaded SF-based film of titanium. The synergic antibacterial mechanism of AgNPs and gentamycin were adhering and accumulation on bacterial cell wall and membrane, disruption of cell wall and membrane, damaging of proteins/nucleic acids, and blocking of the transcription process by binding with 16S rRNA (Zhou et al. 2017). $20-30 \%$ of silk consist of sericin protein comprising 18 amino acids (glycine and aspartic acid as abundant amino acids) with glue-like structure, moisture absorbent, hydrophilic and mechanical stretching properties (Luo and Wang 2016). Tyrosine amino acid in this protein can contribute to AgNPs formation by redox reaction. Wound dressing composed of AgNPs/sericin/PVA illustrated the disruption of cell wall and membrane of $P$. aeruginosa, $S$. aureus, and $E$. coli upon treatment for $12 \mathrm{~h}$ of incubation (Figure 4) (Tao et al. 2019). Growth stages of bacteria displayed a different behavior toward antibacterial agents, wherein the lag phase for $S$. aureus $(16 \mathrm{~h})$ and $E$. coli $(12 \mathrm{~h})$ was delayed for AgNPs-sericin/PVA films prepared by UV-assisted method (He et al. 2017).

\section{Conclusion}

Toxicity of AgNPs towards eukaryotic cells is a main side effect for the applications of these MNPs in medicinal field. Many investigators have presented assorted materials in combination with AgNPs to increase the biocompatibility of MNPs under physiological conditions. Among these, polymers specifically biopolymers have garnered more attraction owing to their desirable biocompatibility, bioavailability, weak antigenicity, and biodegradability properties. Bacterial and fungal infections are one of the most critical complications that delay the wound healing specifically in chronic infectious wounds such as DFU. Inhibition of bacterial colonization, promising swelling and porosity properties, absorption ability of blood secretions, and moist environment can be delivered by the polymer-based wound 
dressing. Compared to other wound dressings based on hyaluronic acid/cationized dextran and quercetin/duck's feet collagen/hydroxyapatite, NCs of OSA-PDA-AgNPs demonstrated more porosity of $77.30 \%$. In this review, antibacterial and wound healing activities of important applicable biopolymers including cellulose, chitosan, collagen, alginate, hyaluronic acid, silk fibroin, sericin, CAR, pectin, keratin, starch, and CD in combination with AgNPs are deliberated to acquire a clear viewpoint of these materials and their NMs forms in suitable micro and nanoformulations. Accordingly, each biopolymer can contribute to different stage of wound healing for example chitosan can increase antibacterial activities of AgNPs in infected chronic wounds as well as the application of collagen in wound bandages can attract fibroblast cells and promote deposition of new collagen fibers to the wound area. Cationic charge of chitosan is critical for interaction of this polymer with teichoic acids and lipoteichoic acids (containing phosphate groups) linked to respectively the peptidoglycan and the underlying plasma membrane in Gram-positive bacteria. This function can synergize the antibacterial mechanisms of AgNPs (oxidative stress, increased cell permeability, pore formation in cell envelope, blocking of electron transport chain, cell wall and membrane disruption, nucleic acid and enzymes damage) at low and safe concentrations compared to pure AgNPs. In the case of applicable wound dressing, the mechanical and chemo-physical properties of scaffold to achieve suitable water-retaining, tensile strength, and promotion cell growth properties can be efficiently attained by cellulose and its derivatives. Cellulose derivatives including HPMC and EC showed high physical stability and durability of AgNPs for 3 months period owing to contribution of $\mathrm{C}-\mathrm{O}$ and $\mathrm{OH}$ groups in NPs synthesis and stabilization. Furthermore, a higher ability for internalization of cellulose functionalized AgNPs into bacteria cells was found for HPMC, EC, and MC derivatives.

As an example of protein polymer, collagen has a vital role in acceleration of hemostasis phase, fibroblasts migration along a connective tissue matrix, and nucleation process in wound area. Therefore, the formulation of several polymers is more efficient relative to the combination of one polymer with AgNPs, wherein, in some cases, synthetic polymers can be tremendously helpful to increase the mechanical strength of wound dressings.

\section{Declarations}

\section{Funding}

Not applicable.

\section{Ethics approval}

This study has not reported the results of studies involving humans and/or animals

\section{Consent to participate}

Not applicable.

\section{Consent for publication}


Not applicable.

\section{Conflict of interest}

The authors declare no competing financial interest.

\section{References}

1. Abdelgawad AM, El-Naggar ME, Elsherbiny DA, Ali S, Abdel-Aziz MS, Abdel-Monem YK (2020) Antibacterial carrageenan/cellulose nanocrystal system loaded with silver nanoparticles, prepared via solid-state technique. Journal of Environmental Chemical Engineering 8:104276. doi:https://doi.org/10.1016/j.jece.2020.104276

2. Abdellatif AAH, Alturki HNH, Tawfeek HM (2021) Different cellulosic polymers for synthesizing silver nanoparticles with antioxidant and antibacterial activities. Sci Rep 11:84. doi:10.1038/s41598-02079834-6

3. Afewerki S, Sheikhi A, Kannan S, Ahadian S, Khademhosseini A (2019) Gelatin-polysaccharide composite scaffolds for 3D cell culture and tissue engineering: Towards natural therapeutics. Bioengineering translational medicine 4:96-115

4. Ahmad T, Ismail A, Ahmad SA, Khalil KA, Kumar Y, Adeyemi KD et al (2017) Recent advances on the role of process variables affecting gelatin yield and characteristics with special reference to enzymatic extraction: A review. Food Hydrocolloids 63:85-96. doi:https://doi.org/10.1016/j.foodhyd.2016.08.007

5. Alavi M (2019) Modifications of microcrystalline cellulose (MCC), nanofibrillated cellulose (NFC), and nanocrystalline cellulose (NCC) for antimicrobial and wound healing applications. e-Polymers 19:103-119

6. Alavi M, Jabari E, Jabbari E (2020) Functionalized carbon-based nanomaterials and quantum dots with antibacterial activity: a review. Expert Review of Anti-infective Therapy: 1-10, doi:10.1080/14787210.2020.1810569

7. Alavi M, Karimi N (2019) Biosynthesis of Ag and Cu NPs by secondary metabolites of usnic acid and thymol with biological macromolecules aggregation and antibacterial activities against multi drug resistant (MDR) bacteria. Int J Biol Macromol 128:893-901. doi:https://doi.org/10.1016/j.ijbiomac.2019.01.177

8. Alavi M, Karimi N (2020) Hemoglobin self-assembly and antibacterial activities of bio-modified Ag$\mathrm{MgO}$ nanocomposites by different concentrations of Artemisia haussknechtii and Protoparmeliopsis muralis extracts. Int J Biol Macromol 152:1174-1185. doi:https://doi.org/10.1016/j.ijbiomac.2019.10.207

9. Alavi M, Karimi N, Valadbeigi T (2019) Antibacterial, Antibiofilm, Antiquorum Sensing, Antimotility, and Antioxidant Activities of Green Fabricated Ag, $\mathrm{Cu}, \mathrm{TiO} 2, \mathrm{ZnO}$, and Fe3O4 NPs via 
Protoparmeliopsis muralis Lichen Aqueous Extract against Multi-Drug-Resistant Bacteria. ACS Biomaterials Science Engineering 5:4228-4243. doi:10.1021/acsbiomaterials.9b00274

10. Alavi M, Nokhodchi A (2020) Antimicrobial and Wound Treatment Aspects of Micro-and Nanoformulations of Carboxymethyl, Dialdehyde, and TEMPO-Oxidized Derivatives of Cellulose: Recent Advances. Macromol Biosci 20:1900362

11. Alavi M, Rai M (2019) Recent progress in nanoformulations of silver nanoparticles with cellulose, chitosan, and alginic acid biopolymers for antibacterial applications. Appl Microbiol Biotechnol 103:8669-8676

12. Alavi M, Rai M (2020) Topical delivery of growth factors and metal/metal oxide nanoparticles to infected wounds by polymeric nanoparticles: an overview. Expert Review of Anti-infective Therapy 18:1021-1032. doi:10.1080/14787210.2020.1782740

13. Alemzadeh E, Oryan A, Mohammadi AA (2020) Hyaluronic acid hydrogel loaded by adipose stem cells enhances wound healing by modulating IL-1 $\beta$, TGF- $\beta 1$, and bFGF in burn wound model in rat. Journal of Biomedical Materials Research Part B: Applied Biomaterials 108:555-567

14. Alipour R, Khorshidi A, Shojaei AF, Mashayekhi F, Moghaddam MJM (2019) Skin wound healing acceleration by Ag nanoparticles embedded in PVA/PVP/Pectin/Mafenide acetate composite nanofibers. Polym Testing 79:106022. doi:https://doi.org/10.1016/j.polymertesting.2019.106022

15. Anuduang A, Loo YY, Jomduang S, Lim SJ, Wan Mustapha WA (2020) Effect of thermal processing on physico-chemical and antioxidant properties in mulberry silkworm (Bombyx mori L.) powder. Foods 9:871

16. Athira GK, Jyothi AN, Vishnu VR (2018) Water Soluble Octenyl Succinylated Cassava StarchCurcumin Nanoformulation With Enhanced Bioavailability and Anticancer Potential. Starch-Stärke 70: 1700178

17. Bastos Araruna F, Oliveira Sousa Araruna F, Lima Alves Pereira LP, Aranha Brito MC, Veras Quelemes P, de Araújo-Nobre AR et al (2020) Green syntheses of silver nanoparticles using babassu mesocarp starch (Attalea speciosa Mart. ex Spreng.) and their antimicrobial applications. Environmental Nanotechnology Monitoring Management 13:100281. doi:https://doi.org/10.1016/j.enmm.2019.100281

18. Bundjaja V, Santoso SP, Angkawijaya AE, Yuliana M, Soetaredjo FE, Ismadji S et al (2021) Fabrication of cellulose carbamate hydrogel-dressing with rarasaponin surfactant for enhancing adsorption of silver nanoparticles and antibacterial activity. Materials Science Engineering: C 118:111542. doi:https://doi.org/10.1016/j.msec.2020.111542

19. Celebioglu A, Topuz F, Yildiz ZI, Uyar T (2019) One-step green synthesis of antibacterial silver nanoparticles embedded in electrospun cyclodextrin nanofibers. Carbohyd Polym 207:471-479. doi:https://doi.org/10.1016/j.carbpol.2018.12.008

20. Chen S, Wu J, Tang Q, Xu C, Huang Y, Huang D et al (2020) Nano-micelles based on hydroxyethyl starch-curcumin conjugates for improved stability, antioxidant and anticancer activity of curcumin. Carbohydrate polymers 228:115398 
21. Dill V, Mörgelin M (2020) Biological dermal templates with native collagen scaffolds provide guiding ridges for invading cells and may promote structured dermal wound healing. Int Wound J 17:618630. doi:10.1111/iwj.13314

22. Ding C, Tian M, Feng R, Dang Y, Zhang M (2020) Novel Self-Healing Hydrogel with Injectable, pHResponsive, Strain-Sensitive, Promoting Wound-Healing, and Hemostatic Properties Based on Collagen and Chitosan. ACS Biomaterials Science Engineering 6:3855-3867

23. Diniz FR, Maia RCAP, Rannier L, Andrade LN, Chaud V, da Silva M CF, et al (2020) Silver nanoparticlescomposing alginate/gelatine hydrogel improves wound healing in vivo. Nanomaterials 10:390

24. Divakar P, Yin K, Wegst UGK (2019) Anisotropic freeze-cast collagen scaffolds for tissue regeneration: How processing conditions affect structure and properties in the dry and fully hydrated states. J Mech Behav Biomed Mater 90:350-364

25. El-Aassar MR, Ibrahim OM, Fouda MMG, El-Beheri NG, Agwa MM (2020) Wound healing of nanofiber comprising Polygalacturonic/Hyaluronic acid embedded silver nanoparticles: In-vitro and in-vivo studies. Carbohyd Polym 238:116175. doi:https://doi.org/10.1016/j.carbpol.2020.116175

26. El-Fiqi A, Lee JH, Lee E-J, Kim H-W (2013) Collagen hydrogels incorporated with surface-aminated mesoporous nanobioactive glass: improvement of physicochemical stability and mechanical properties is effective for hard tissue engineering. Acta biomaterialia 9:9508-9521

27. Esparza Y, Ullah A, Boluk Y, Wu J (2017) Preparation and characterization of thermally crosslinked poly (vinyl alcohol)/feather keratin nanofiber scaffolds. Materials Design 133:1-9

28. Ezhilarasu H, Vishalli D, Dheen ST, Bay B-H, Srinivasan DK (2020) Nanoparticle-Based Therapeutic Approach for Diabetic Wound Healing. Nanomaterials 10:1234

29. Feroz S, Muhammad N, Ranayake J, Dias G (2020) Keratin - Based materials for biomedical applications. Bioactive materials 5:496-509. doi:10.1016/j.bioactmat.2020.04.007

30. García-Ríos V, Ríos-Leal E, Robledo D, Freile-Pelegrin Y (2012) Polysaccharides composition from tropical brown seaweeds. Phycological Research 60:305-315

31. Ge L, Xu Y, Li X, Yuan L, Tan H, Li D et al (2018) Fabrication of Antibacterial Collagen-Based Composite Wound Dressing. ACS Sustainable Chemistry Engineering 6:9153-9166. doi:10.1021/acssuschemeng.8b01482

32. Goel A, Meher MK, Gupta P, Gulati K, Pruthi V, Poluri KM (2019) Microwave assisted K-carrageenan capped silver nanocomposites for eradication of bacterial biofilms. Carbohyd Polym 206:854-862. doi:https://doi.org/10.1016/j.carbpol.2018.11.033

33. Gou L, Xiang M, Ni X (2020) Development of wound therapy in nursing care of infants by using injectable gelatin-cellulose composite hydrogel incorporated with silver nanoparticles. Mater Lett 277:128340. doi:https://doi.org/10.1016/j.matlet.2020.128340

34. Graça MFP, Miguel SP, Cabral CSD, Correia IJ (2020) Hyaluronic acid-Based wound dressings: A review. Carbohyd Polym 241:116364. doi:https://doi.org/10.1016/j.carbpol.2020.116364

35. Guo T, Yang X, Deng J, Zhu L, Wang B, Hao S (2019) Keratin nanoparticles-coating electrospun PVA nanofibers for potential neural tissue applications. Journal of Materials Science: Materials in 
Medicine 30:9

36. Han Y, Lv S (2019) Synthesis of chemically crosslinked pullulan/gelatin-based extracellular matrixmimetic gels. Int J Biol Macromol 122:1262-1270

37. He H, Cai R, Wang Y, Tao G, Guo P, Zuo H et al (2017) Preparation and characterization of silk sericin/PVA blend film with silver nanoparticles for potential antimicrobial application. Int J Biol Macromol 104:457-464. doi:https://doi.org/10.1016/j.ijbiomac.2017.06.009

38. Jiang Y, Wang D, Li F, Li D, Huang Q (2020) Cinnamon essential oil Pickering emulsion stabilized by zein-pectin composite nanoparticles: characterization, antimicrobial effect and advantages in storage application. Int J Biol Macromol 148:1280-1289

39. Kaczmarek B, Nadolna K, Owczarek A (2020) Chapter 6 - The physical and chemical properties of hydrogels based on natural polymers. In: Chen $Y$ (ed) Hydrogels Based on Natural Polymers. Elsevier, pp 151-172

40. Li K, Guan G, Zhu J, Wu H, Sun Q (2019) Antibacterial activity and mechanism of a laccase-catalyzed chitosan-gallic acid derivative against Escherichia coli and Staphylococcus aureus. Food Control 96:234-243. doi:https://doi.org/10.1016/j.foodcont.2018.09.021

41. Liang L, Hou T, Ouyang Q, Xie L, Zhong S, Li P et al (2020) Antimicrobial sodium alginate dressing immobilized with polydopamine-silver composite nanospheres. Composites Part B: Engineering 188:107877. doi:https://doi.org/10.1016/j.compositesb.2020.107877

42. Lim Y-S, Ok Y-J, Hwang S-Y, Kwak J-Y, Yoon S (2019) Marine collagen as a promising biomaterial for biomedical applications. Marine drugs 17:467

43. Lomelí-Marroquín D, Medina Cruz D, Nieto-Argüello A, Vernet Crua A, Chen J, Torres-Castro A et al (2019) Starch-mediated synthesis of mono- and bimetallic silver/gold nanoparticles as antimicrobial and anticancer agents. Int J Nanomed 14:2171-2190. doi:10.2147/IJN.S192757

44. Luo Y, Wang T (2016) Chapter 9 - Pharmaceutical and Cosmetic Applications of Protein By-Products. In: Singh G, Dhillon (eds) Protein Byproducts. Academic Press, pp 147-160

45. Mercado-Mercado G, Laura A, Alvarez-Parrilla E (2020) Effect of pectin on the interactions among phenolic compounds determined by antioxidant capacity. J Mol Struct 1199:126967

46. Moghadam A, ljaz M, Asim MH, Mahmood A, Jelkmann M, Matuszczak B et al (2018) Non-ionic thiolated cyclodextrins - the next generation. Int J Nanomed 13:4003-4013. doi:10.2147/IJN.S153226

47. Mohamadi Zahedi S, Mansourpanah Y (2018) Construction of chitosan-carboxymethyl $\beta$ cyclodextrin silver nanocomposite hydrogel to improve antibacterial activity. Plast Rubber Compos 47:273-281. doi:10.1080/14658011.2018.1475166

48. Mohammed H, Kumar A, Bekyarova E, Al-Hadeethi Y, Zhang X, Chen M et al (2020) Antimicrobial Mechanisms and Effectiveness of Graphene and Graphene-Functionalized Biomaterials. A Scope Review. [10.3389/fbioe.2020.00465]. Frontiers in Bioengineering and Biotechnology 8: 465

49. Nanda SS, Yi DK, Kim K (2016) Study of antibacterial mechanism of graphene oxide using Raman spectroscopy. Sci Rep 6:28443. doi:10.1038/srep28443

Page 14/19 
50. Palazzo C, Trapani G, Ponchel G, Trapani A, Vauthier C (2017) Mucoadhesive properties of low molecular weight chitosan-or glycol chitosan-and corresponding thiomer-coated poly (isobutylcyanoacrylate) core-shell nanoparticles. Eur J Pharm Biopharm 117:315-323

51. Pallavicini P, Arciola CR, Bertoglio F, Curtosi S, Dacarro G, D'Agostino A et al (2017) Silver nanoparticles synthesized and coated with pectin: An ideal compromise for anti-bacterial and antibiofilm action combined with wound-healing properties. J Colloid Interface Sci 498:271-281. doi:https://doi.org/10.1016/j.jcis.2017.03.062

52. Pandey S, Do JY, Kim J, Kang M (2020) Fast and highly efficient catalytic degradation of dyes using K-carrageenan stabilized silver nanoparticles nanocatalyst. Carbohyd Polym 230:115597. doi:https://doi.org/10.1016/j.carbpol.2019.115597

53. Patil S, Dhyani V, Kaur T, Singh N (2020) Spatiotemporal Control over Cell Proliferation and Differentiation for Tissue Engineering and Regenerative Medicine Applications Using Silk Fibroin Scaffolds. ACS Applied Bio Materials

54. Pietrucha K (2005) Changes in denaturation and rheological properties of collagen-hyaluronic acid scaffolds as a result of temperature dependencies. Int J Biol Macromol 36:299-304

55. Pooresmaeil M, Namazi H (2019) Preparation and characterization of polyvinyl alcohol/ $\beta-$ cyclodextrin/GO-Ag nanocomposite with improved antibacterial and strength properties. Polym Adv Technol 30:447-456

56. Pouget C, Dunyach-Remy C, Pantel A, Schuldiner S, Sotto A, Lavigne J-P (2020) Biofilms in Diabetic Foot Ulcers: Significance and Clinical Relevance. Microorganisms 8:1580

57. Ran X, Du Y, Wang Z, Wang H, Pu F, Ren J et al (2017) Hyaluronic acid-templated Ag nanoparticles/graphene oxide composites for synergistic therapy of bacteria infection. ACS Appl Mater Interfaces 9:19717-19724

58. Shao J, Wang B, Li J, Jansen JA, Walboomers XF, Yang F (2019) Antibacterial effect and wound healing ability of silver nanoparticles incorporation into chitosan-based nanofibrous membranes. Materials Science Engineering: C 98:1053-1063. doi:https://doi.org/10.1016/j.msec.2019.01.073

59. Tao G, Cai R, Wang Y, Liu L, Zuo H, Zhao P et al (2019) Bioinspired design of AgNPs embedded silk sericin-based sponges for efficiently combating bacteria and promoting wound healing. Materials Design 180:107940. doi:https://doi.org/10.1016/j.matdes.2019.107940

60. Taran M, Rad M, Alavi M (2016) Characterization of Ag nanoparticles biosynthesized by Bacillus sp. HAl4 in different conditions and their antibacterial effects. Journal of Applied Pharmaceutical Science 6:094-099

61. Tian B, Hua S, Liu J (2020) Cyclodextrin-based delivery systems for chemotherapeutic anticancer drugs: A review. Carbohyd Polym 232:115805. doi:https://doi.org/10.1016/j.carbpol.2019.115805

62. Usman A, Hussain Z, Riaz A, Khan AN (2016) Enhanced mechanical, thermal and antimicrobial properties of poly(vinyl alcohol)/graphene oxide/starch/silver nanocomposites films. Carbohyd Polym 153:592-599. doi:https://doi.org/10.1016/j.carbpol.2016.08.026 
63. Valentine ME, Kirby BD, Withers TR, Johnson SL, Long TE, Hao Y et al (2020) Generation of a highly attenuated strain of Pseudomonas aeruginosa for commercial production of alginate. Microb Biotechnol 13:162-175

64. Wan Y, Yang S, Wang J, Gan D, Gama M, Yang Z et al (2020) Scalable synthesis of robust and stretchable composite wound dressings by dispersing silver nanowires in continuous bacterial cellulose. Composites Part B: Engineering 199:108259. doi:https://doi.org/10.1016/j.compositesb.2020.108259

65. Wang J, Xu J (2020) Effects of Topical Insulin on Wound Healing: A Review of Animal and Human Evidences. Diabetes, metabolic syndrome and obesity: targets and therapy 13: 719-727, doi:10.2147/DMSO.S237294

66. Wang Y, Li P, Xiang P, Lu J, Yuan J, Shen J (2016) Electrospun polyurethane/keratin/AgNP biocomposite mats for biocompatible and antibacterial wound dressings. Journal of Materials Chemistry B 4:635-648

67. Wei Y, Zhang J, Zhou Y, Bei W, Li Y, Yuan Q et al (2017) Characterization of glabridin/hydroxypropyl$\beta$-cyclodextrin inclusion complex with robust solubility and enhanced bioactivity. Carbohydrate polymers 159:152-160

68. Yang X-R, Zhao Y-Q, Qiu Y-T, Chi C-F, Wang B (2019) Preparation and characterization of gelatin and antioxidant peptides from gelatin hydrolysate of skipjack tuna (Katsuwonus pelamis) bone stimulated by in vitro gastrointestinal digestion. Mar Drugs 17:78

69. You C, Li Q, Wang X, Wu P, Ho JK, Jin R et al (2017) Silver nanoparticle loaded collagen/chitosan scaffolds promote wound healing via regulating fibroblast migration and macrophage activation. Sci Rep 7:10489. doi:10.1038/s41598-017-10481-0

70. Zhang H, Peng M, Cheng T, Zhao P, Qiu L, Zhou J et al (2018) Silver nanoparticles-doped collagenalginate antimicrobial biocomposite as potential wound dressing. Journal of Materials Science 53:14944-14952

71. Zhou W, Jia Z, Xiong P, Yan J, Li Y, Li M et al (2017) Bioinspired and biomimetic AgNPs/gentamicinembedded silk fibroin coatings for robust antibacterial and osteogenetic applications. ACS Appl Mater Interfaces 9:25830-25846

\section{Figures}

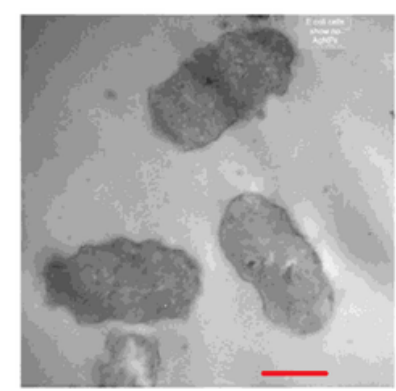

A

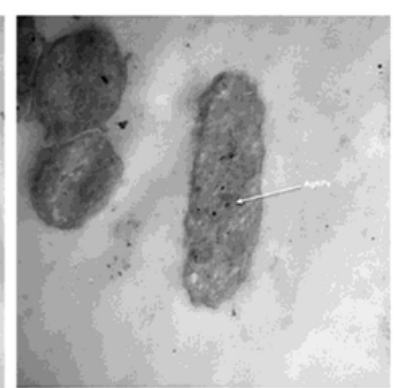

B

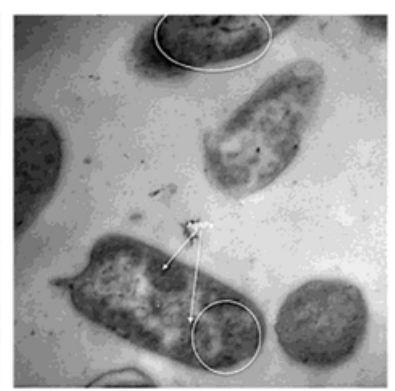

C

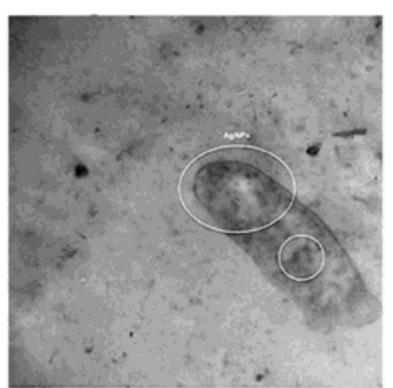

D

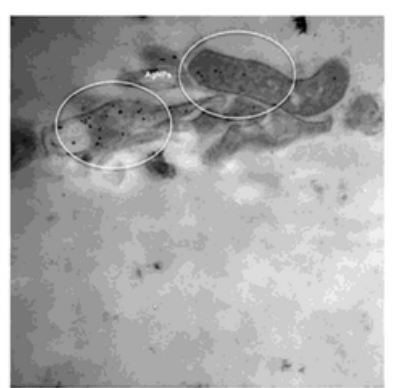

E 
Figure 1

Different internalization of AgNPs into E. coli: A) Control, B) PEG-AgNPs, C) MC-AgNPs, D) EC-AgNPs, and HPMC-AgNPs; scale bar is $500 \mathrm{~nm}$ for all images (Abdellatif et al. 2021).
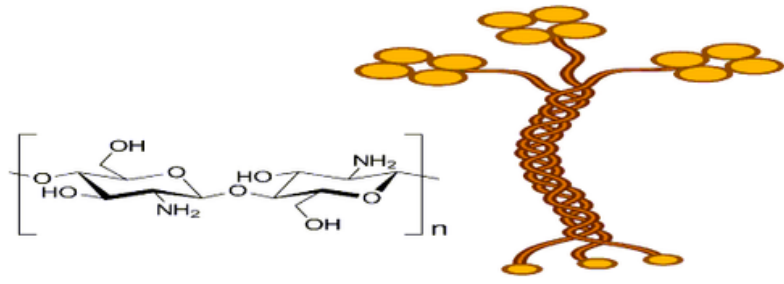

a)
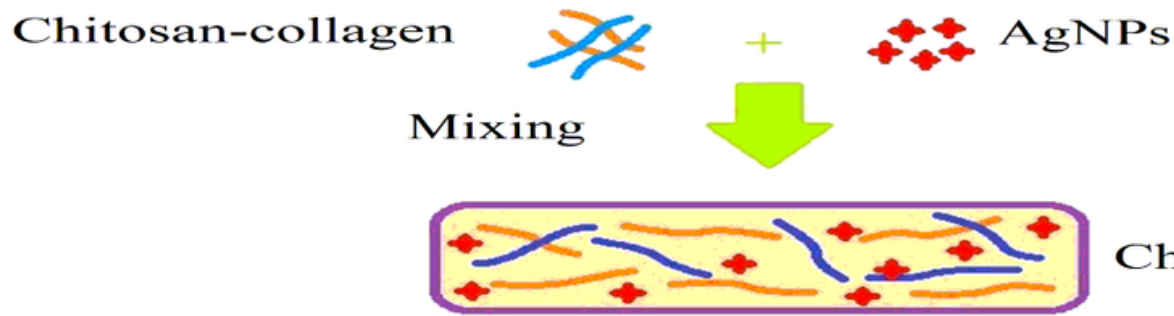

Chitosan-collagen-AgNPs

Implantation on damaged skin

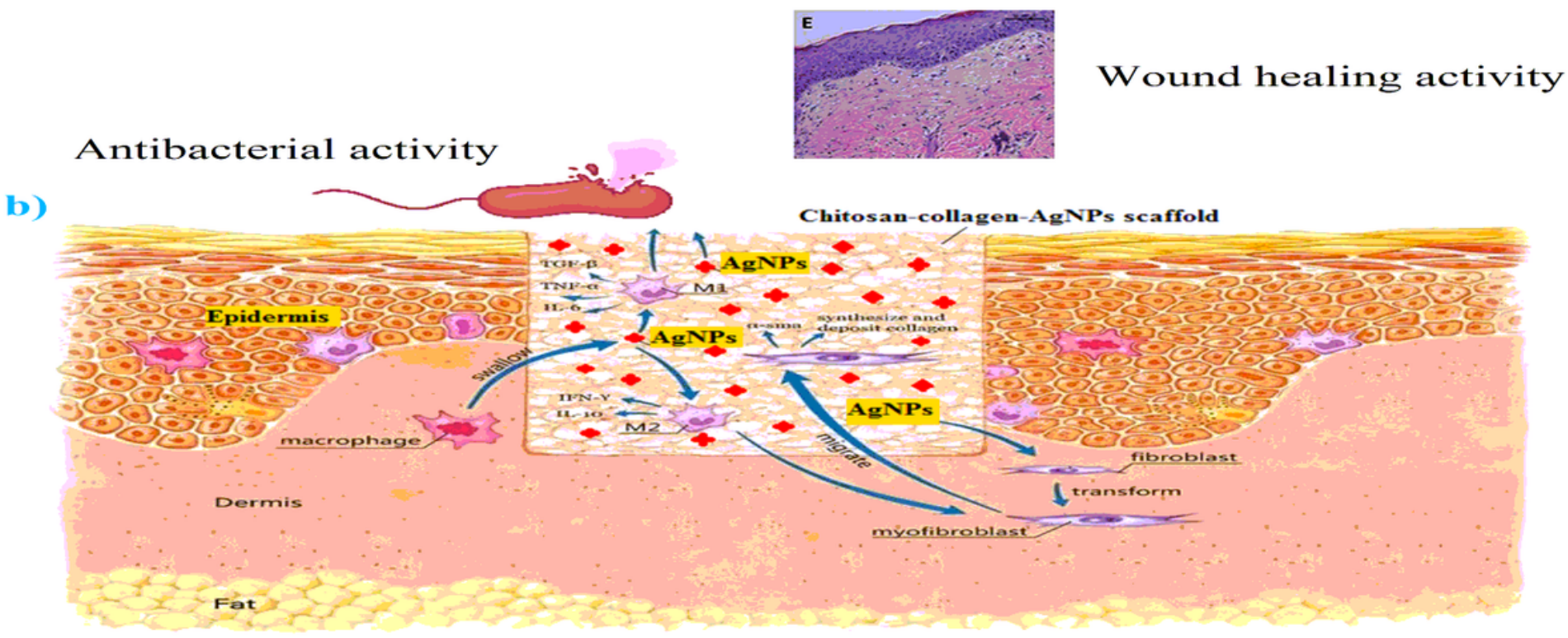

Figure 2

The possible mechanisms for accelerated cutaneous wound healing by scaffold of chitosan-collagenAgNPs; tumor necrosis factor-a (TNF-a), transforming growth factor- $\beta$ (TGF- $\beta$ ), interleukin 6 (IL-6), interleukin 10 (IL-10), interferon gamma (IFN- - ), a-smooth muscle actin (a-SMA), classical macrophage activation (M1) and alternative macrophage activation (M2) (Adapted and modified from (You et al. 2017) and BioRender.com). 


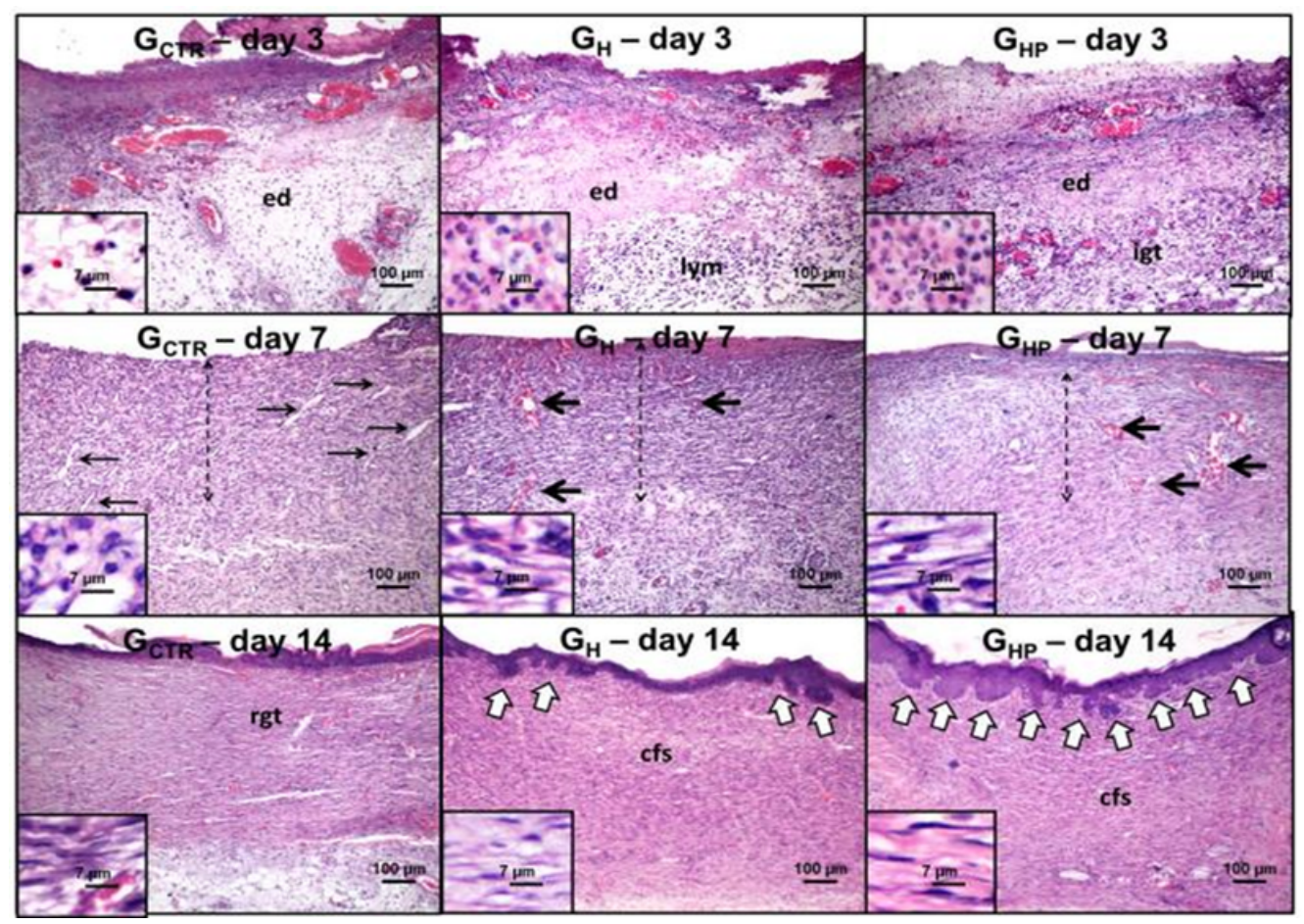

(a)

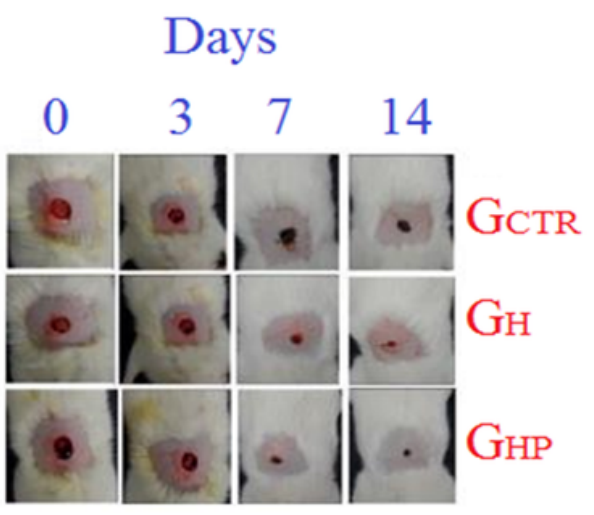

(b)

Figure 3

Histological sections (a) and images of wound closure (b) showing a striking wound healing in the period of 14 days of experiment. (Intense edema (ed), the lymphocyte-rich infiltrate (lym) and immature granulation tissue (igt), a cellular primary fibrous scar (cfs); groups of hydrogel with AgNPs 4 mM AgNO3 (GHP), hydrogel sodium alginate/gelatin (80:20) (GH), and control (GCTR)) (Adapted and modified from (Diniz et al. 2020)).

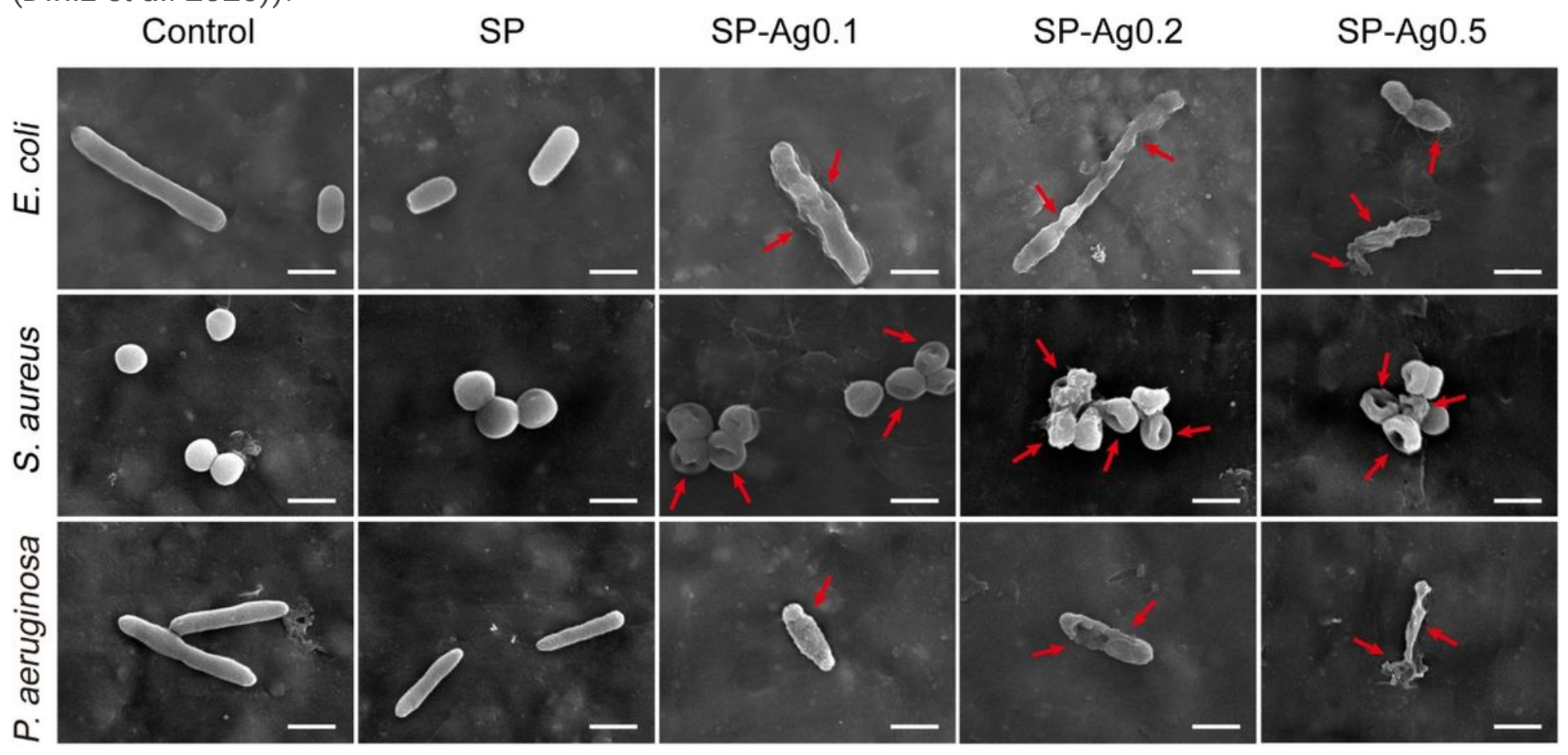

Figure 4 
Morphology alteration of bacterial cell envelope under treatment of sericin/PVA (SP) and NCs of SP with different concentrations of AgNPs (0.1, 0.2, and $0.5 \mathrm{mM} / \mathrm{L}$ of AgNO3). Complete cell wall deformation and disruption are mainly observable in the higher concentration of AgNPs (Tao et al. 2019). 